\title{
Synchrony analysis: application in early diagnosis, staging and prognosis of multiple sclerosis
}

\author{
Zahra Ghanbari and Shahriar Gharibzadeh* \\ Department of Biomedical Engineering, Amirkabir University of Technology, Tehran, Iran \\ *Correspondence: gharibzadeh@aut.ac.ir
}

Edited by:

Tobias Alecio Mattei, Ohio State University, USA

Reviewed by:

Al-Rahim Abbasali Tailor, The Ohio State University Wexner Medical Center, USA

Keywords: multiple sclerosis (MS), synchrony, early diagnosis, staging, prognosis, prediction

Multiple Sclerosis (MS) is an autoimmune disease caused by degeneration of the myelin sheath of large diameter fibers in the central nervous system. This will cause deficits in the conducting properties of nerves and also affect electrical signaling. As a result, in MS patients, nerve conduction will be slower than normal (Kandel et al., 2000).

Neural synchrony has been of great interest in neuroscience recently. In signal processing, synchrony refers to quantifying similarity, coherence or correlation among signals and could be measured using a variety of methods (Dauwels et al., 2010). Neural synchrony represents how synchronous the neurons are firing (Vialatte et al., 2008). It is proven that synchrony is an important feature of brain signals. Many neurological diseases are accompanied by abnormalities in neural synchrony (Dauwels et al., 2008).

For example, loss of synchrony among brain signals has been observed in disorders such as Parkinson's and Alzheimer's disease $(\mathrm{AD})$ and was used for the purpose of diagnosis. On the other hand, increasing synchrony has been reported in disorders such as epileptic seizures (Vialatte et al., 2009).

Since perturbation in electrical signaling and slowing of nerve conduction are common among MS and the aforementioned diseases, it brings up the idea of using synchrony for MS as well. In addition, previous works on MS have reported loss of connectivity and synchronous function among different parts of patients' brains. It should be mentioned that most of the previous works were concentrated on the cognitive impairments caused by the disease, and they applied their methods on MEG signals (Arrondo et al., 2009; Hardmeier et al., 2012).

The other point which should be noted is that although MRI and ERP are both common tools in MS diagnosis and follow up, definite diagnosis cannot be made based on these criteria individually. In addition, MRI needs to be repeated (Greenberg et al., 2009; Longo et al., 2012) and it is not affordable and available in many situations. So, we should try to find a reliable solution.

According to the aforementioned points, we believe that recording electrical brain signals (particularly EEG and ERP) and calculating local and global synchrony among their channels may provide us with an individual tool for diagnosing MS. Actually, the idea we put forward is using calculated synchrony indices for the purpose of detection, classification and prediction on electrical brain signals. Of course, the previous results which investigated connectivity and synchronous function of brain parts support our idea (Arrondo et al., 2009; Hardmeier et al., 2012).

The proposed idea may also help us to detect MS in early stages. Additionally, we believe as impairments will increase by progression of the disease, synchrony measures may have significant differences in different stages of the disease. So, they could be useful for staging of the disease as well.

We also propose measuring synchrony among brain signals in the onset periods. It seems that there should be a correlation between the changes in synchrony measures and disease prognosis. In better words, based on the calculated synchrony indices, we can predict the trend of the disease. This would provide us with a clearer perspective of the possible efficiency of different management modalities (including medical and surgical). Additionally, based on the potential level of neural dyssynchrony the proposed idea can be useful in order to assess the efficiency of the selected treatments for both the patient and the physician. Surely experimental evaluations are needed to validate our hypothesis.

\section{REFERENCES}

Arrondo, G., Alegre, M., Sepulcre, J., Iriarte, J., Artieda, J., and Villoslada, P. (2009). Abnormalities in brain synchronization are correlated with cognitive impairment in multiple sclerosis. Mult. Scler. 15, 509-516. doi: 10.1177/1352458508101321

Dauwels, J., Vialatte, F., and Cichocki, A. (2008). "Quantifying statistical synchrony: algorithms and applications to brain data analysis and early prediction of Alzheimer's disease," in Proceedings of the 3rd INFORMS Workshop on Data Mining and Health Informatics (DM-HI 2008), eds J. Li, D. Aleman, and R. Sikora.

Dauwels, J., Vialatte, F., Musha, T., and Cichocki, A (2010). A comparative study of synchrony measures for the early diagnosis of Alzheimer's disease based on EEG. Neuroimage 49, 668-693. doi: 10.1016/j.neuroimage.2009.06.056

Greenberg, D. A., Aminoff, M., and Simon, M. R. (2009). Clinical Neurology. New York, NY: McGraw Hill.

Hardmeier, H., Schoonheim, M., Geurts, J., Hillebrand, A., Polman, C., Barkhof, F., et al. (2012). Cognitive dysfunction in early multiple sclerosis: altered centrality derived from resting-state functional connectivity using magneto-encephalography. PLoS ONE 7:e42087. doi: 10.1371/journal.pone.0042087

Kandel, E. R., Schwartz, J. H., and Jessell, T. M. (2000). Principles of Neural Science, 4 th Edn. New York, NY: McGraw-Hill Companies.

Longo, D. L., Fauci, A., Kasper, D., Hauser, S., and Jameson, J. (2012). Harrison's Principles of Internal Medicine. New York, NY: McGraw Hill. 
Vialatte, F., Dauwels, J., Rutkowski, T., and Cichocki, A. (2008). "Measuring neural synchrony by message passing," in Advances in Neural Information Processing Systems (Washington, DC), 361-368.

Vialatte, F., Sole-Casals, J., Dauwels, J., Maurice, M., and Cichocki, A. (2009). Bump time-frequency toolbox: a toolbox for time-frequency oscillatory bursts extraction in electrophysiological signals. BMC Neurosci. 10:46. doi: 10.1186/1471-22 02-10-46
Conflict of Interest Statement: The authors declare that the research was conducted in the absence of any commercial or financial relationships that could be construed as a potential conflict of interest.

Received: 26 April 2014; accepted: 27 June 2014; published online: 21 July 2014.

Citation: Ghanbari Z and Gharibzadeh S (2014)

Synchrony analysis: application in early diagnosis, staging and prognosis of multiple sclerosis. Front. Comput. Neurosci. 8:73. doi: 10.3389/fncom.2014.00073
This article was submitted to the journal Frontiers in Computational Neuroscience.

Copyright () 2014 Ghanbari and Gharibzadeh. This is an open-access article distributed under the terms of the Creative Commons Attribution License (CC BY). The use, distribution or reproduction in other forums is permitted, provided the original author(s) or licensor are credited and that the original publication in this journal is cited, in accordance with accepted academic practice. No use, distribution or reproduction is permitted which does not comply with these terms. 